\title{
Chopping effect observed at cathodic arc initiation
}

André Anders, Senior Member, IEEE

\begin{abstract}
Chopping of current and induction of high voltage spikes has been observed at the rising edge of cathodic vacuum arcs. It is argued that current chopping at the rising edge is similar to the current chopping effect that is well-know for the arc current approaching current-zero. Current chopping can generate high voltage spikes, and thus cathodic arc equipment should be protected against voltage spikes that exceed the systems nominal voltage by an order of magnitude.
\end{abstract}

\section{Index Terms}

Vacuum arcs, vacuum interrupters, chopping current, voltage spikes 
Cathodic arcs or "vacuum" arcs can be initiated by a variety of techniques. While DC or quasi-continuous arcs often utilize a mechanically moved trigger electrode, pulsed arcs are usually triggered by a high-voltage surface flashover across an insulator that separates the arc cathode from the high-voltage trigger electrode.

An alternative method of arc initiation which uses neither a mechanical electrode nor a very high pulsed voltage was dubbed the "triggerless" method [1]. It is based on explosive formation of a "hot spot" by ohmic heating of a contact point at the interface between the cathode and a conductive coating that is deposited on an insulator touching the cathode. The coating brings the anode potential to the contact point. The only power supply involved is the arc power supply. The coating is regenerated by plasma deposition. The "triggerless" arc initiation technique was successfully demonstrated in our laboratory for over 50 cathode materials and has been implemented in pulsed plasma sources for thin film deposition [1] and in a vacuum arc ion source [2]. In the following we focus on a scheme with "triggerless" arc initiation although the observed chopping effect may also occur with other arc initiation techniques.

The chopping effect has been well studied [3-5] due to its importance for vacuum interrupters. In essence, the vacuum arc develops instabilities and extinguishes when the arc current becomes smaller than a critical value: the current "chops off". The critical current value depends on the cathode material, the cathode surface condition, the electrode configuration, the magnetic field (if present), and the electric circuit parameters. The phenomenon is associated with the plasma-generating mechanism of the arc spot. When the arc current is decreases and approaches current-zero, the number of current-carrying arc spots, or spot fragments [6], decreases, and the amplitude of noise in the arc voltage 
increases. When the arc current is further decreased, the conditions for the ignition of spot fragments deteriorate. For instance, cathode surface heating by ion bombardment decreases. If no new spot fragments are ignited, plasma production and electron emission at cathode spots ceases, the current transport is interrupted, and the arc extinguishes. The phenomenon can be harmful if it occurs at relatively high critical chopping current because high voltage spikes can be induced that can be dangerous to personnel and equipment. Special electrode materials such as $\mathrm{CuW}$ and $\mathrm{CuCr}$ have been developed showing very low chopping currents.

The chopping effect is usually observed for decreasing arc current. In this publication, we report on the observation of the chopping effect with increasing current after arc initiation. As pointed out by a reviewer, the effect is not really new but often observed with AC vacuum arc interrupters after current-zero [7].

Figure 1 shows the electrical schematic of the experiment. Cathodic arcs are initiated simply by applying the charging voltage of a $0.5 \Omega$ pulse-forming-network to the arc electrodes (360 V in the example shown). The resistor $R_{t l} \sim 1 \mathrm{k} \Omega$ in Fig.1 symbolizes the resistance of the conducting path between the anode and cathode that is used for "triggerless" arc initiation. The cathode was an aluminum rod surrounded by an aluminum-coated ceramic tube (plasma source of the mini-gun type [8]). The vacuum pressure was about $10^{-4} \mathrm{~Pa}$. The arc current, measured by a wide-band current transformer of sensitivity $0.01 \mathrm{~V} / \mathrm{A}$, shows a current rise-time that is determined by the inductance of the arc circuit. In the example shown in Figure 2, the thyristor's (SCR) switching time of about $1 \mu$ s is manifested by small voltage variations while the current is still close to zero. The current flows initially through the conducting path, limited by the resistance of the conducting path. For most arc pulses, the current rise is smooth. However, some arc pulses exhibit an instability as show in Figure 
2. The arc current is still small when the instability occurs, indicating that the underlying physical reasons are similar to what is known for the chopping effect at decreasing arc current. A statistical test of several hundred arc pulses showed that the instability occurred for about $10 \%$ of the pulses and, if present, occurred always at currents less than $60 \mathrm{~A}$.

Although more studies are needed to prove that the mechanism of the chopping effect observed with increasing current is indeed identical to the mechanism for decreasing current, high voltage spikes are induced in both cases. The amplitude of the spikes can exceed the charging voltage by a factor $3-5$, and sometimes even higher spikes are observed. Power supplies and other equipment are often not adequately protected. Failure of equipment by overvoltage has been observed. Noteworthy, this type of failure occurred when operating with relatively low charging voltage, in agreement with the interpretation that the instability leading to current chopping and voltage generation is more likely to occur at low arc current. I would like to thank Ian Brown for his continued support and encouragement. This work was supported by the U.S. Department of Energy, under Contract No. DE-AC0376SF00098. 


\section{Figure Captions}

Figure 1 Simplified electrical schematic of the experiment. The pulse-forming-network had an impedance of $0.5 \Omega$, and the charging voltage could be varied up to $1 \mathrm{kV}$. The resistor $R_{\mathrm{tl}}$ represents the resistance of the conducting path used for "triggerless" arc initiation (typically $\sim 1 \mathrm{k} \Omega$ ). The locations of current and voltage measurements are indicated.

Figure 2 Example of current and voltage measurements showing an instability that may be associated with the chopping effect. Note that the voltage spikes are about $1.4 \mathrm{kV}$ although the charging voltage is only $360 \mathrm{~V}$. 


\section{References}

[1] A. Anders, I. G. Brown, R. A. MacGill, and M. R. Dickinson, ""Triggerless" triggering of vacuum arcs," J. Phys. D: Appl. Phys., vol. 31, pp. 584-587, 1998.

[2] A. Anders, J. Schein, and N. Qi, "Pulsed vacuum-arc ion source operated with a "triggerless" arc initiation method," Rev. Sci. Instrum., vol. 71, pp. 827-829, 2000.

[3] R. P. P. Smeets, "The origin of current chopping in vacuum arcs," IEEE Trans. Plasma Sci., vol. 17, pp. 303-310, 1989.

[4] R. P. P. Smeets, "Experimental characterization of arc instabilities and their effect on current chopping in low-surge vacuum interrupters," IEEE Trans. Plasma Sci., vol. 20, pp. 439-446, 1992.

[5] A. Yamamoto, T. Okutomi, M. Homma, and M. Okawa, "Instability of vacuum arc in Ag-Cu contact alloys,” IEEE Trans. Plasma Sci., vol. 21, pp. 463-468, 1993.

[6] B. Jüttner, "The dynamics of arc cathode spots in vacuum," J. Phys. D: Appl. Phys., vol. 28, pp. 516-522, 1995.

[7] R. P. P. Smeets, W. J. van der Ven, K. Watanabe, and M. Homma, "Vacuum arc lifetime extension at small gaplength: beneficial for low-surge circuit interruption," presented at Eleventh Int. Conf. on Gas Discharges and Their Applications, vol. 1, Tokyo, Japan, 1995, pp. 82-85.

[8] R. A. MacGill, M. R.Dickinson, A. Anders, O. R. Monteiro, and I. G. Brown, "Streaming metal plasma generation by vacuum arc plasma guns," Rev. Sci. Instrum., vol. 69, pp. 801-803, 1998. 


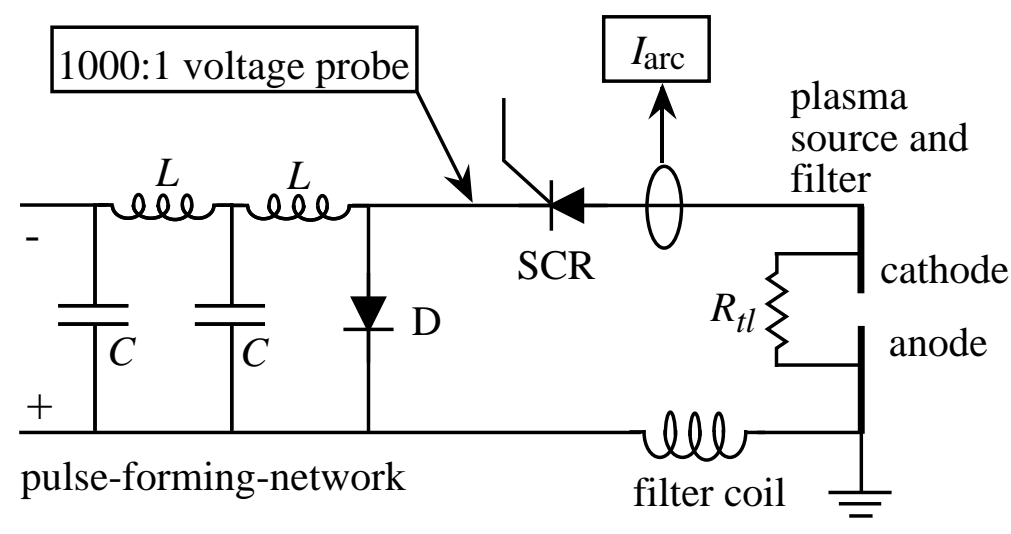

Figure 1 


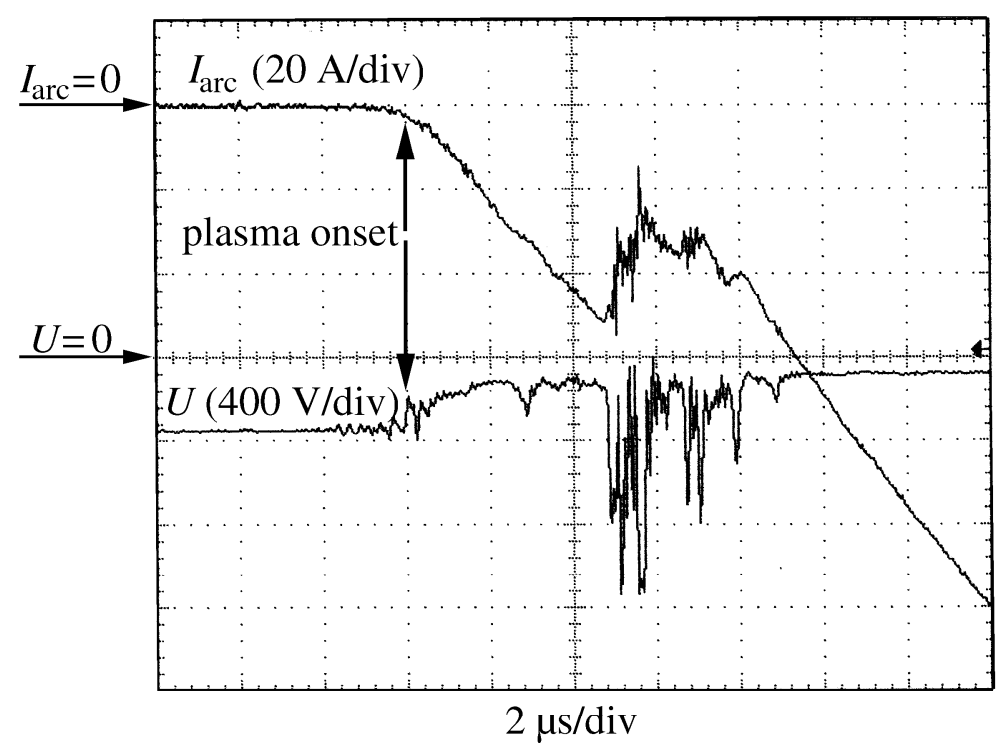

Fig. 2 